\title{
Reasons for non-recruitment of eligible patients to a randomised controlled trial of secondary prevention after intracerebral haemorrhage: observational study
}

Amy E. Maxwell ${ }^{1}$, Mary Joan MacLeod ${ }^{2}$, Anu Joyson², Sharon Johnson ${ }^{3}$, Hawraman Ramadan ${ }^{4}$, Ruth Bellfield ${ }^{4}$, Anthony Byrne ${ }^{5}$, Caroline McGhee ${ }^{5}$, Anthony Rudd ${ }^{6}$, Fiona Price ${ }^{7}$, Evangelos Vasileiadis ${ }^{8}$, Melinda Holden 8 , Jonathan Hewitt ${ }^{9}$, Michael Carpenter ${ }^{10}$, Ann Needle ${ }^{10}$, Stacey Valentine ${ }^{11}$, Farzana Patel ${ }^{12}$, Frances Harrington ${ }^{13}$, Paul Mudd ${ }^{14}$, Hedley Emsley ${ }^{15}$, Bindu Gregary ${ }^{15}$, Ingrid Kane ${ }^{16}$, Keith Muir ${ }^{17}$, Divya Tiwari ${ }^{18}$, Peter Owusu-Agyei ${ }^{19}$, Natalie Temple ${ }^{19}$, Lakshmanan Sekaran ${ }^{20}$, Suzanne Ragab ${ }^{21}$, Timothy England ${ }^{22}$, Amanda Hedstrom²2, Phil Jones ${ }^{23}$, Sarah Jones ${ }^{23}$, Mandy Doherty ${ }^{24,25}$, Mark O. McCarron ${ }^{25}$, David L. Cohen ${ }^{26}$, Sharon Tysoe ${ }^{27}$

and Rustam Al-Shahi Salman ${ }^{*}$

\begin{abstract}
Background: Recruitment to randomised prevention trials is challenging, not least for intracerebral haemorrhage $(\mathrm{ICH})$ associated with antithrombotic drug use. We investigated reasons for not recruiting apparently eligible patients at hospital sites that keep screening logs in the ongoing REstart or STop Antithrombotics Randomised Trial (RESTART), which seeks to determine whether to start antiplatelet drugs after ICH.

Method: By the end of May 2015, 158 participants had been recruited at 108 active sites in RESTART. The trial coordinating centre invited all sites that kept screening logs to submit screening log data, followed by one reminder. We checked the integrity of data, focused on the completeness of data about potentially eligible patients and categorised the reasons they were not randomised.
\end{abstract}

Results: Of 108 active sites, 39 (36\%) provided usable screening log data over a median of ten (interquartile range $=5-13)$ months of recruitment per site. During this time, sites screened 633 potentially eligible patients and randomised 53 (8\%) of them. The main reasons why 580 patients were not randomised were: 43 (7\%) patients started anticoagulation, 51 (9\%) patients declined, 148 (26\%) patients' stroke physicians were not uncertain about using antiplatelet drugs, 162 (28\%) patients were too unwell and 176 (30\%) patients were not randomised due to other reasons.

Conclusion: RESTART recruited $\sim 8 \%$ of eligible patients. If more physicians were uncertain about the therapeutic dilemma that RESTART is addressing, RESTART could have recruited up to four times as many participants. The trial coordinating centre continues to engage with physicians about their uncertainty.

Trial registration: EU Clinical Trials, EudraCT 2012-003190-26. Registered on 3 July 2012.

Keywords: Stroke, Intracerebral haemorrhage, Recruitment, Screening log

\footnotetext{
* Correspondence: Rustam.Al-Shahi@ed.ac.uk

${ }^{1}$ Centre for Clinical Brain Sciences, University of Edinburgh, Chancellor's

Building, 49 Little France Crescent, Edinburgh EH16 4SB, UK

Full list of author information is available at the end of the article
} 


\section{Background}

Research into methods to boost recruitment has been identified as the highest priority for randomised controlled trials (RCTs) methodological research in the UK [1]. The Lancet's series on 'Research: Increasing Value and Reducing Waste' identified under-recruitment to RCTs as a major source of inefficiency in the conduct of applied clinical research [2]. Slow recruitment is particularly inefficient because it delays the delivery of research and inflates its costs by increasing the number of staff and sites or by extending the amount and duration of funding required.

This problem has not been small - a review of 114 RCTs funded by MRC or HTA in the UK in 1994-2002 found that less than one-third recruited their original target within the time originally specified and around one-third were given extensions to achieve their target [3]. A re-investigation of this in 2002-2008 found that almost half of the RCTs did not recruit their originally specified target sample size and nearly half of the RCTs received an extension of some kind [4], which was only a marginal improvement. Recruitment is jeopardised by many factors including restrictive eligibility criteria and inefficient methods for approaching potential participants $[5,6]$. Screening logs may help inform the representativeness of a RCT [7], but they may also be informative for understanding why eligible patients are not recruited $[8,9]$.

Stroke due to intracerebral haemorrhage (ICH) affects $\sim 10,000$ adults in the UK each year. Within one month of $\mathrm{ICH} \sim 40 \%$ of patients die and more than half the survivors are dependent [10]. Achieving large sample sizes in $\mathrm{ICH}$ RCTs is therefore challenging, especially when protocols specify short time windows for recruitment after $\mathrm{ICH}$ onset and include many eligibility criteria [5]. Many ICH RCTs have studied acute and rehabilitation interventions, but only one published RCT has investigated an intervention for secondary prevention; PROGRESS recruited 611 patients with $\mathrm{ICH}$ at 172 hospital sites in ten countries over 30 months in 1995-1997 [11, 12].

The REstart or STop Antithrombotics Randomised Trial (RESTART, ISRCTN71907627 www.restarttrial.org) is an ongoing RCT comparing policies of restarting versus avoiding antiplatelet drugs for secondary prevention after ICH. RESTART aimed to recruit 720 participants over two years (from May 2013 to May 2015) based on recent epidemiological data $[5,13]$. RESTART has implemented as many as possible of the strategies that have been shown to maximise recruitment $[5,14,15]$ including: minimising the number of eligibility criteria, maximising the time window for recruiting patients after $\mathrm{ICH}$ onset, and having an open design where participants know which treatment they are receiving. In addition, the Chief Investigator has used qualitative methods with
Principal Investigators at all active sites to identify and overcome barriers to clinician recruitment activity, as recommended by a systematic review [16].

Based on pilot data in an international collaboration [17], 20\% of all ICH patients (2\% of all strokes) should be potentially eligible for RESTART (although these data were unable to take account of patients' functional status and a recent hospital-based study found that only $15 \%$ of patients form the Lund Stroke Registry were potentially eligible for RESTART [18]). We completed a quantitative investigation of recruitment estimates given by sites when being activated to RESTART in February 2015, and at the time of this investigation 101 sites expected to have recruited 741 patients, but they had in fact recruited 135 (18\%). By the end of May 2015, 108 hospital sites were active and 158 participants had been randomised in RESTART (22\% of the recruitment target of 720 participants).

Therefore, we sought to identify the main reasons for fewer participants being recruited than expected.

\section{Methods}

To investigate why potentially eligible patients were not being randomised in RESTART we requested sites provide us anonymised screening logs. Although sites had not been asked to specifically keep screening logs for the trial, the trial coordinating centre were aware that some centres kept them for their stroke patients as that was their practice or they were required to by their $R \& D$ department. By gathering these valuable data, we could analyse what the leading reasons for not recruiting potentially eligible patients were.

The RESTART trial coordinating centre invited all sites by email on 7 May 2015, asking them if they could provide anonymised logs (if they kept them) containing a list of their patients with $\mathrm{ICH}$ who had been screened at their hospital site since becoming active in RESTART along with the outcome e.g. 'randomised', 'not eligible', 'patient declined,' 'doctor not uncertain', etc. We sent a reminder email on 22 May 2015 to maximise responses by the deadline of 31 May 2015.

The RESTART eligibility criteria are as follows. Inclusion criteria: patient age $\geq 18$ years; spontaneous primary or secondary $\mathrm{ICH}$; patient had taken antithrombotic $\operatorname{drug}(\mathrm{s})$ for the prevention of vaso-occlusive disease before $\mathrm{ICH}$ onset; randomisation more than $24 \mathrm{~h}$ after $\mathrm{ICH}$ onset; patient and their doctor are uncertain about whether to start or avoid antiplatelet drugs; patient is registered with a general practitioner; brain imaging that first diagnosed the $\mathrm{ICH}$ is available; participant or representative consent. Exclusion criteria: $\mathrm{ICH}$ due to preceding trauma or haemorrhagic transformation of ischaemic stroke; patient is taking an anticoagulant drug following $\mathrm{ICH}$; patient is pregnant, breastfeeding or of childbearing age and 
not taking contraception; patient and carer unable to understand spoken or written English.

We asked RESTART hospital sites with available screening log data to record: the date the patient was screened; whether the patient's diagnosis of $\mathrm{ICH}$ was within the two months before screening; whether the patient was screened as an inpatient or outpatient; and the outcome of the screening (ineligible, eligible and randomised, or eligible but not randomised). We categorised reasons for non-recruitment of potentially eligible patients (according to the eligibility criteria above) as follows, and assumed that investigators had recorded the leading reason if there had been more than one:

- Patient declined

- Doctor not uncertain

- Started anticoagulant

- Unwell / end of life / died

- Other

We reviewed the data integrity, completeness and consistency of each submission of screening log data. We collated data in an Excel spreadsheet and recorded key information from each site's submission, including: the time period of screening log data collection, the number of patients screened, the number of patients that were randomised to RESTART, whether patients were ineligible and, if eligible, the reasons why they were not recruited (categorised as above).

\section{Results}

Of the 108 active RESTART sites emailed to invite them to provide their screening logs, 44 (41\%) provided data, but we were unable to use data provided by five sites, leaving data from 39 (36\%) of RESTART hospital sites.

The 39 sites that returned screening logs recruited 58 patients between May 2013 and May 2015 (1.49 patients per site) and the 69 sites that did not provide screening logs recruited 100 patients during the same (1.45 patients per site), providing no evidence of bias among the sites included in our analyses of screening logs.

The data from the 39 sites with screening logs covered a median of ten (interquartile range $=5-13$ ) months per site. During this time, sites randomised 53 participants and also screened 580 patients who appeared eligible but were not randomised (ratio 1:11) (Fig. 1).

The main reasons why the 580 patients were not randomised were: 43 (7\%) patients started anticoagulation; 51 (9\%) patients declined; 148 (26\%) had stroke physicians who were not uncertain about using antiplatelet drugs; 162 (28\%) patients were too unwell; and 176 (30\%) patients were not randomised due to other reasons, for example, lived out of catchment area, transferred to a different hospital, lost to follow-up, enrolled

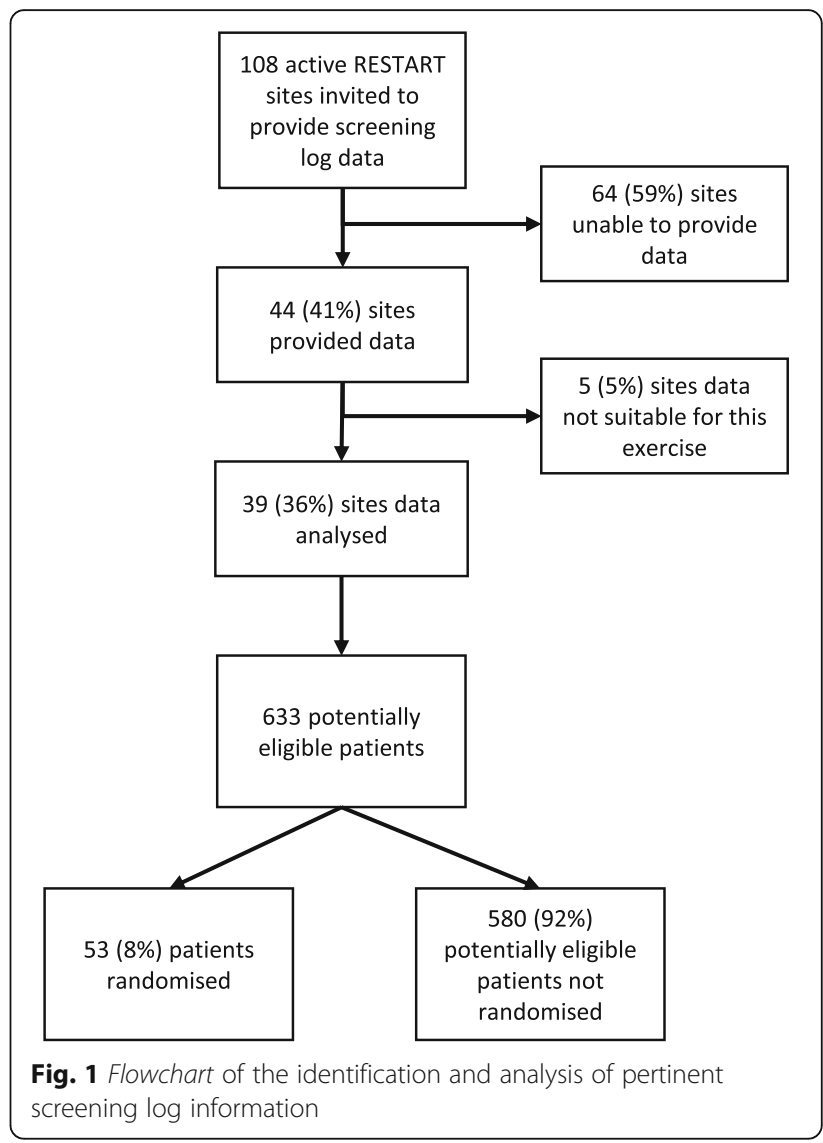

in another drug trial, still under review, consented but not recruited or no clear reason was provided (Fig. 2).

\section{Discussion}

Screening log data demonstrate that $8 \%$ of potentially eligible patients were recruited and randomised to the RESTART trial of secondary prevention after stroke due to $\mathrm{ICH}$. The two leading single reasons for eligible patients not being recruited was that they were too unwell/

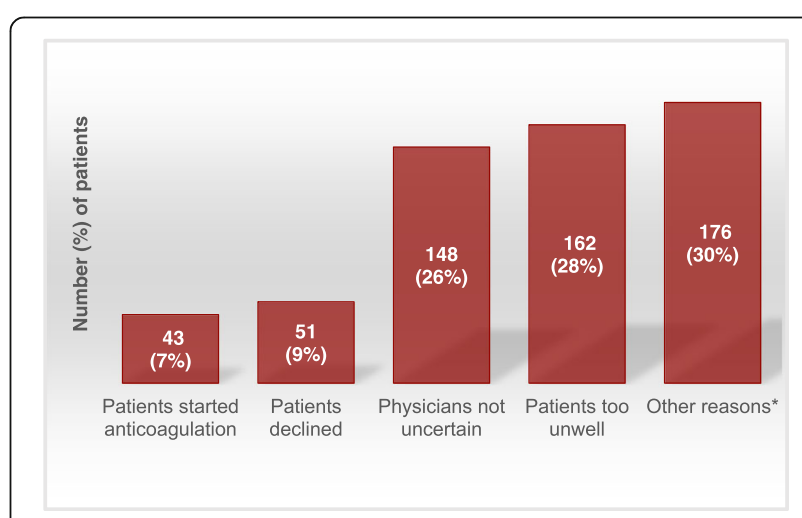

Fig. 2 Graph showing reasons for 580 potentially eligible patients not being randomised 
disabled and that physicians were not uncertain despite the clear shortage of evidence to inform this therapeutic dilemma in the available literature $[19,20]$.

This study has some strengths. The RESTART trial reflects contemporary clinical practice and trial recruitment at a large number of hospitals in the UK. RESTART is the only ongoing RCT of secondary prevention after $\mathrm{ICH}$ in the UK. We gave all sites the opportunity to participate, checked the data that we received and used a standardised approach to categorising reasons for non-recruitment.

This study has some weaknesses. RESTART did not mandate sites to keep screening logs - so they were only available at a minority of sites - but used those that were available because sites recorded screening activity to monitor their research activity. Sites returned data in a variety of formats and collected data over slightly differing time periods because there was variation in the time at which they became active in the collaboration. We had to omit some data due to their quality or completeness.

Screening logs appear useful for identifying the leading reasons why eligible patients are not recruited to a RCT. These data can inform future RCTs of pharmacological secondary prevention $\mathrm{RCTs}$ after $\mathrm{ICH}$, in which $\sim 8 \%$ of eligible participants may be recruited, although we cannot exclude the possibility that this proportion is interventionspecific. Physician certainty, despite the lack of rigorous evidence to inform therapeutic decisions, remains one of the major, potentially modifiable barriers to recruiting larger proportions of eligible patients.

Due to under-recruitment, RESTART's funder kindly granted an extension of recruitment until 31 May 2018.

\section{Conclusion}

Screening log data can be useful for identifying reasons for low recruitment of eligible patients to a RCT. Approximately $8 \%$ of eligible patients were recruited to the RESTART trial. During the period of this screening log analysis, if some physicians had not been certain about whether to start antiplatelet drugs after ICH for 148 potentially eligible patients, RESTART could have almost quadrupled the number of participants recruited from 53 to 201.

\section{Abbreviations}

Cl: Chief Investigator; ICH: Intracerebral haemorrhage; RCT: Randomised controlled trial; RESTART: REstart or STop Antithrombotics Randomised Trial

\footnotetext{
Acknowledgements

We thank Heather Gow (Aberdeen Royal Infirmary), Ashraf Ahmed (Barnsley Hospital NHS Foundation Trust), Manohar Kini and Deborah Walstow (Doncaster Royal Infirmary), Sagal Kullane (Guys \& St Thomas, London), Colin Jenkins (Hereford County Hospital), Bella Richard (Nevill Hall Hospital, Abergavenny), Jessica Whiteman (Nevill Hall Hospital, Abergavenny \& Ystrad Mynach Hospital, Newport), Harinath Chandrashekar and Emily Tenbruck (Ninewells Hospital \& Medical School, Dundee), Julie Ball (Pinderfields Hospital, Wakefield), Prasad Siddegowda and Tracey Dobson (Queen Alexandra Hospital, Portsmouth), Neetish Goorah (Royal Blackburn Hospital), Ali James (Royal Cornwall Hospital) Angela Bowring (Royal Devon \& Exeter
}

Hospital), Joanna Breeds (Royal Sussex County Hospital, Brighton), John Paterson and Kerry Deighton (Scarborough Hospital), Nicola Day (South Glasgow University Hospital), Jo Bell and Barbara Longland (The Royal Bournemouth Hospital), David Wilson and Belinda Wroath (Ulster Hospital), Mahesh Dhakal and Susan Clayton (University Hospital North Durham), Arindam Kar and Beth Hazel (Charing Cross Hospital, London), Janet Putterhill and Rachel Gallifent (Epsom General Hospital), Aparna Pusalkar and Kelly Chan (Lister Hospital, Stevenage), Santhosh Subramonian (Peterborough City Hospital), Frances Justin and Meena Chauhan (Luton \& Dunstable NHSFT University Hospital), Judith Dube (Poole Hospital), Nabarun Sengupta and Maria Metiu (Worthing Hospital), James Mcllmoyle and Carol Jeffs (Victoria Hospital, Blackpool), Claire Duggan and Abigail Hynes (Bronglais Hospital, Aberystwyth), Breffni Keegan and Jim Kelly (South West Acute Hospital, Enniskillen), Mushiya Mpelembue (Northwick Park Hospital, Harrow), Ursula Schulz and Rachel Teal (John Radcliffe Hospital, Oxford), Youssif Abousleiman, Sally Collins and Linda Still (East Surrey Hospital, Redhill), Devesh Sinha (Southend University Hospital NHS Foundation Trust) for providing screening log data, the British Heart Foundation for funding RESTART and The Academic and Clinical Central Office for Research and Development (ACCORD) for sponsoring RESTART.

Funding

RESTART is supported by British Heart Foundation Special Project (SP/12/2/ 29422) and Project (PG/14/50/30891) grants.

Availability of data and materials

The data that support the findings of this study are available from the corresponding author upon reasonable request.

\section{Authors' contributions}

RA-SS designed the study with input from AEM, supervised data analysis and revised the first draft of this report. AEM collected and analysed data and wrote the first draft of the report. MM, AJ, SJ, HR, RB, AB, CM, AR, FP, EV, MH, $J H, M C, A N, S V, F P, F H, P M, H E, B G, I K, K M, D T, P O-A, N T, L S, S R, T E, A H, P J, S J$, MD, MOM, DLC and ST collected data. All authors reviewed and approved the final draft of the manuscript.

\section{Competing interests}

RA-SS is the Chief Investigator of RESTART. The remaining authors declare that they have no competing interests.

\section{Consent for publication}

Not applicable for this report of analyses of anonymous screening log data.

\section{Ethics approval and consent to participate}

The Scotland A research ethics committee approved the RESTART and all its participating sites on 2 November 2012 (Ref. 12/SS/0138). The RESTART protocol and study conduct comply with the Medicines for Human Use (Clinical Trials) Regulations 2004 and any relevant amendments. All participants, or their nearest relative, provide consent to participate in RESTART, but informed consent was not required for this report of analyses of anonymous screening log data.

\section{Publisher's Note}

Springer Nature remains neutral with regard to jurisdictional claims in published maps and institutional affiliations.

\section{Author details}

${ }^{1}$ Centre for Clinical Brain Sciences, University of Edinburgh, Chancellor's Building, 49 Little France Crescent, Edinburgh EH16 4SB, UK. ${ }^{2}$ Aberdeen Royal Infirmary, Aberdeen, UK. ${ }^{3}$ Barnsley Hospital NHS Foundation Trust, Barnsley, UK. ${ }^{4}$ Bradford Royal Infirmary, Bradford, UK. ${ }^{5}$ Forth Valley Royal Hospital, Larbert, UK. ${ }^{6}$ Guys \& St Thomas, London, UK. ${ }^{7}$ Hereford County Hospital, Hereford, UK. ${ }^{8}$ Hillingdon Hospital, Uxbridge, UK. ${ }^{9}$ Ystrad Mynach Hospital, Newport, UK. ${ }^{10}$ Pinderfields Hospital, Wakefield, UK. ${ }^{11}$ Queen Alexandra Hospital, Portsmouth, UK. ${ }^{12}$ Royal Blackburn Hospital, Blackburn, UK. ${ }^{13}$ Royal Cornwall Hospital, Cornwall, UK. ${ }^{14}$ Royal Devon \& Exeter Hospital, Exeter, UK. ${ }^{15}$ Royal Preston Hospital, Preston, UK. ${ }^{16}$ Royal Sussex County Hospital, Brighton, UK. ${ }^{17}$ South Glasgow University Hospital, Glasgow, UK. ${ }^{18}$ The Royal Bournemouth Hospital, Bournemouth, UK. ${ }^{19}$ Peterborough City Hospital, Peterborough, UK. ${ }^{20}$ Luton \& Dunstable NHSFT University Hospital, Luton, UK. 
${ }^{21}$ Poole Hospital, Poole, UK. ${ }^{22}$ Derby Royal Hospital, Derby, UK. ${ }^{23}$ Bronglais Hospital, Aberystwyth, UK. ${ }^{24}$ South West Acute Hospital, Enniskillen, UK. ${ }^{25}$ Altnagelvin Hospital, Londonderry, UK. ${ }^{26}$ Northwick Park Hospital, Harrow, UK. ${ }^{27}$ Southend University Hospital NHS Foundation Trust, Southend-on-Sea, UK.

Received: 4 October 2016 Accepted: 18 March 2017

Published online: 05 April 2017

\section{References}

1. Tudur Smith C, Hickey H, Clarke M, Blazeby J, Williamson P. The trials methodological research agenda: results from a priority setting exercise. Trials. 2014;15:32.

2. Al-Shahi Salman R, Beller E, Kagan J, Hemminki E, Phillips RS, Savulescu J, et al. Increasing value and reducing waste in biomedical research regulation and management. Lancet. 2014;383:176-85.

3. Campbell MK, Snowdon C, Francis D, Elbourne D, McDonald AM, Knight R, et al. Recruitment to randomised trials: strategies for trial enrollment and participation study. The STEPS study. Health Technol Assess. 2007; 1 1:iii, ix-105.

4. Sully BG, Julious SA, Nicholl J. A reinvestigation of recruitment to randomised, controlled, multicenter trials: a review of trials funded by two UK funding agencies. Trials. 2013;14:166.

5. Fonville AF, Samarasekera N, Hutchison A, Perry D, Roos YB, Al-Shahi Salman R. Eligibility for randomized trials of treatments specifically for intracerebral hemorrhage: community-based study. Stroke. 2013;44:2729-34.

6. McDonald AM, Knight RC, Campbell MK, Entwistle VA, Grant AM, Cook JA, et al. What influences recruitment to randomised controlled trials? A review of trials funded by two UK funding agencies. Trials. 2006;7:9.

7. Slieker FJ, Kompanje EJ, Murray GD, Ohman J, Stocchetti N, Teasdale SG, et al. Importance of screening logs in clinical trials for severe traumatic brain injury. Neurosurgery. 2008;62:1321-8. discussion 8-9.

8. St Germain D, Denicoff AM, Dimond EP, Carrigan A, Enos RA, Gonzalez MM, et al. Use of the National Cancer Institute Community Cancer Centers Program screening and accrual log to address cancer clinical trial accrual. J Oncol Pract. 2014;10:e73-80.

9. Duchesne GM, Woo HH, Howell D, Kaimakamis M, Kemp E. Tribulations of a prostate cancer trial - lessons learned from TOAD, a Cancer Council Victoria and Transtasman Radiation Oncology Group trial. J Med Imaging Radiat Oncol. 2010:54:508-11.

10. van Asch CJ, Luitse MJ, Rinkel GJ, van der Tweel I, Algra A, Klijn CJ. Incidence, case fatality, and functional outcome of intracerebral haemorrhage over time, according to age, sex, and ethnic origin: a systematic review and meta-analysis. Lancet Neurol. 2010;9:167-76.

11. PROGRESS Collaborative Group. Randomised trial of a perindopril-based blood-pressure-lowering regimen among 6,105 individuals with previous stroke or transient ischaemic attack. Lancet. 2001;358:1033-41.

12. Chapman N, Huxley R, Anderson C, Bousser MG, Chalmers J, Colman S, et al. Effects of a perindopril-based blood pressure-lowering regimen on the risk of recurrent stroke according to stroke subtype and medical history: the PROGRESS Trial. Stroke. 2004;35:116-21.

13. Samarasekera N, Fonville A, Lerpiniere C, Farrall AJ, Wardlaw JM, White PM, et al. Influence of intracerebral hemorrhage location on incidence, characteristics, and outcome: population-based study. Stroke. 2015;46:361-8.

14. Watson JM, Torgerson DJ. Increasing recruitment to randomised trials: a review of randomised controlled trials. BMC Med Res Methodol. 2006;6:34.

15. Treweek S, Lockhart P, Pitkethly M, Cook JA, Kjeldstrom M, Johansen M, et al. Methods to improve recruitment to randomised controlled trials: Cochrane systematic review and meta-analysis. BMJ Open. 2013;3:e002360

16. Fletcher B, Gheorghe A, Moore D, Wilson S, Damery S. Improving the recruitment activity of clinicians in randomised controlled trials: a systematic review. BMJ Open. 2012;2:e000496.

17. Pasquini M, Charidimou A, van Asch CJ, Baharoglu MI, Samarasekera N, Werring DJ, et al. Variation in restarting antithrombotic drugs at hospital discharge after intracerebral hemorrhage. Stroke. 2014;45:2643-8.

18. Hansen BM, Ullman N, Norrving B, Hanley DF, Lindgren A. Applicability of clinical trials in an unselected cohort of patients with intracerebral hemorrhage. Stroke. 2016;47:2634-7.
19. Flynn RW, MacDonald TM, Murray GD, Doney AS. Systematic review of observational research studying the long-term use of antithrombotic medicines following intracerebral hemorrhage. Cardiovasc Ther. 2010:28:177-84.

20. Al-Shahi Salman R, Dennis MS. Antiplatelet therapy may be continued after intracerebral hemorrhage. Stroke. 2014;45:3149-50.

\section{Submit your next manuscript to BioMed Central and we will help you at every step:}

- We accept pre-submission inquiries

- Our selector tool helps you to find the most relevant journal

- We provide round the clock customer support

- Convenient online submission

- Thorough peer review

- Inclusion in PubMed and all major indexing services

- Maximum visibility for your research

Submit your manuscript at www.biomedcentral.com/submit
) Biomed Central 